\title{
Case report: Delayed spontaneous retroperitoneal haematoma after unilateral total hip replacement
}

\author{
Ana Torres Pérez ${ }^{1 *}$, Mariano Fernández Fairen², Julio Duart Clemente ${ }^{3}$, Javier del Río Alonso ${ }^{3}$, Matías Alfonso Olmos ${ }^{3}$ and Carlos Villas \\ Tome $^{3}$ \\ ${ }^{1}$ Department of Orthopaedics. Santa Lucia's Universitary Hospital, Spain \\ ${ }^{2}$ Department of orthopaedics. University of Navarra. Pamplona, Spain \\ ${ }^{3}$ Instituto del Aparato Locomotor. Barcelona, Spain
}

\begin{abstract}
We present the case of a 59-year-old woman who developed a major spontaneous retroperitoneal haematoma after performing a unilateral total hip arthroplasty. We reviewed the literature to see that a retroperitoneal haematoma is a rare event which can potentially be life-threatening.

In our patient, the abdominal pain instead of being acute, as it happens on the majority of retroperitoneal haematomas, it started at day five postop associated to a sensation of abdominal fullness. An urgent CT was ordered to achieve de diagnose.

A selective angiographic embolization was employed to stop the bleeding. Even if a total hip arthroplasty is a safe procedure, we need to be alert on operated patients with abdominal pain after the surgery and perform a careful evaluation.
\end{abstract}

\section{Introduction}

The annual number of hip prostheses in the world is constantly increasing, representing the second most common surgical procedure [1].

Following a total hip prosthesis, the causes of pain are numerous and of varied origins [2]: first, pain due to the approach, extraprosthetic causes (rachidian pathology tendinomyopathy of the hip abductors), infectious or mechanical aetiologies (failure of anchorage, conflict pathology). Vascular injuries after a total hip replacement (THR) are rare [3].

Haematomas are not described in literature, except in rare cases of compressive haematomas [4]. This complication is classically described as following bad anticoagulation (overdosing) or insufficient haemostasis. It usually appears as a progressive external sciatic popliteal paralysis with deglobulinization.

If it appears early, the differential diagnosis with primary neurological injury without haematoma, most frequently due to an indirect trauma (compression, stretching or intraoperative ischemia), may be problematic.

Pouliot [5] described in 2008 a unique case of major retroperitoneal haematoma which occurred three years and a half after surgery and was attributed to an iliopsoas/cup conflict.

Spontaneous retroperitoneal bleeding is a rare event which can potentially be life-threatening [6]. The majority of cases if they are not related to trauma, surgery or puncture; are due to ruptured renal lesions (such as angiomyolipomas [7-9]; however, vascular disorders [10,11] and anticoagulation therapy [12-18], may also be the underlying cause. Sometimes is not possible to find an abnormality responsible for the bleeding [19]
Renal tumours account for $58 \%$ to $79 \%$ of cases of non-traumatic retroperitoneal haemorrhage $[7,20]$. Presenting symptoms and clinical signs depend on the degree and duration of bleeding and therefore may vary significantly.

We present a case of major spontaneous retroperitoneal bleeding in a patient after a unilateral total hip replacement (THR)

\section{Patient and method}

\section{Case report}

A 59 years old woman $(80 \mathrm{~kg}, 142 \mathrm{~cm}$, BMI 39.7) was admitted for a right hip prosthesis because of a severe coxarthrosis. She was under pain while being sitting or walking. Her medical history consisted in diabetes mellitus type II well controlled with oral antidiabetics (metformin and glimepiride).

The preoperative blood test showed a haemoglobin of $15.3 \mathrm{~g} \mathrm{dL}^{-1}$, platelet count $412 \times 10^{9}$, prothrombin time (PT) of $97.7 \%$, international normalised ratio (INR) of 1.0, activated partial thromboplastin time of 36.1 seconds (references value 23 - 33) and a normal renal function. There was no existing coagulation disorder.

The hip prosthesis was performed under spinal anaesthesia. The puncture was performed via posterior and medial approach, at the level L2-L3, using an $80 \mathrm{~mm}, 24$-gauge pencil point tip. Three attempts were needed to accomplish the block. After the spinal puncture the patient was placed in left decubitus lateral position. Prior to skin incision 2

Correspondence to: Ana Torres, M.D, PhD, Calle del Carmen, 59-15 B 30201 Cartagena-Murcia, Spain; Tel: +34-605302700, E-mail: anatpz@gmail.com

Received: March 12, 2017; Accepted: April 07, 2017; Published: April 10, 2017 
gr. of cefazolin were administered intravenously. We have employed an anterolateral approach (Watson Jones) to implant a cemented cup (PLE $44 \mathrm{~mm}$ ), a ceramic liner and head $(32 \mathrm{~mm}$ ) and a CLS Spotorno uncemented stem (size 5). We didn't use screws to fix the cup. Drainage was left during 48 hours postop to drain the postsurgical haematoma. The patient received low molecular weight heparin injections at preventive dosage ( $60 \mathrm{mg} / 24$ hours) no intraoperative and immediate postoperative complication was seen.

Postoperative control haemoglobin, the day after the surgery was $9.1 \mathrm{~g} \mathrm{dL}^{-1}$, no blood transfusion was needed. In our hospital, we have a transfusion protocol, if the patient has not cardiac problems we don't transfuse till the patient haemoglobine reaches $8 \mathrm{~g} \mathrm{dL}^{-1}$. The range of motion of the operated hip was normal.

At day 5, the patient started with a sensation of abdominal fullness followed by pain. The patient was constipated, and clyster was applied. After the evacuation, the patient was pale, and continued with the sensation of abdominal fullness. She presented pain with the palpation and a mass was noted in the left iliac fossa, but the Blumberg's sign was negative

A new blood test showed a blood loss (haemoglobine $6.8 \mathrm{gr} / \mathrm{dL}$ ) and a moderate leukocytosis $\left(21.1 \times 10^{9} \mathrm{~L}^{-1}\right)$. Under the suspicion of being in front of a diverticulitis we have ordered an urgent CT scan where a large retroperitoneal haematoma with anterior displacement of the left kidney (Figure 1) and diffusion of the haematoma into the left psoas and iliac muscles was seen suspecting an origin of the bleeding on the right lumbar artery (Figure 2). A transfusion of three packed erythrocytes concentrate was performed urgently.

An urgent arteriography using a size 4 French Simmons catheter and a Pigtail 4 French catheter (Cordis, Rhoden, The Netherlands) via the right femoral artery revealed multiple points of bleeding at different branches of the left third lumbar artery (Figure 3A). Selective catheterisation of the left third lumbar artery was undertaken with embolization using 300-500 $\mu \mathrm{m}$ and 500-700 $\mu \mathrm{m}$ multiple coils (Cook, Bjaeverskov, Denmark). Follow-up arteriography showed no further bleeding (Figure 3B).

The patient medical status improved in few days and she was discharged on postoperative day 13 with no further complaints. The patient remained symptom free after 10 months walking without crutches and has stopped taking painkillers.

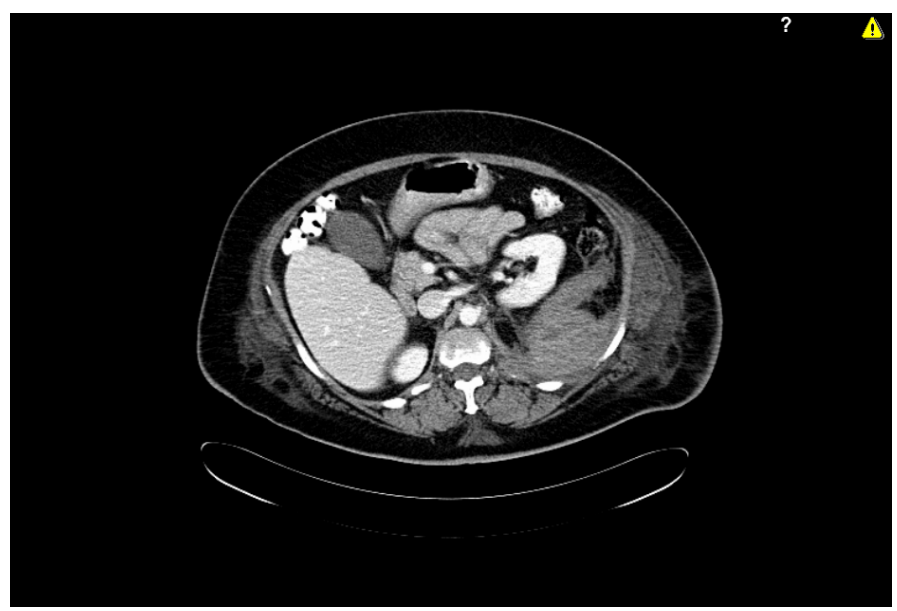

Figure 1. Urgent $\mathrm{CT}$ scan where a large retroperitoneal haematoma with anterior displacement of the left kidney was seen.

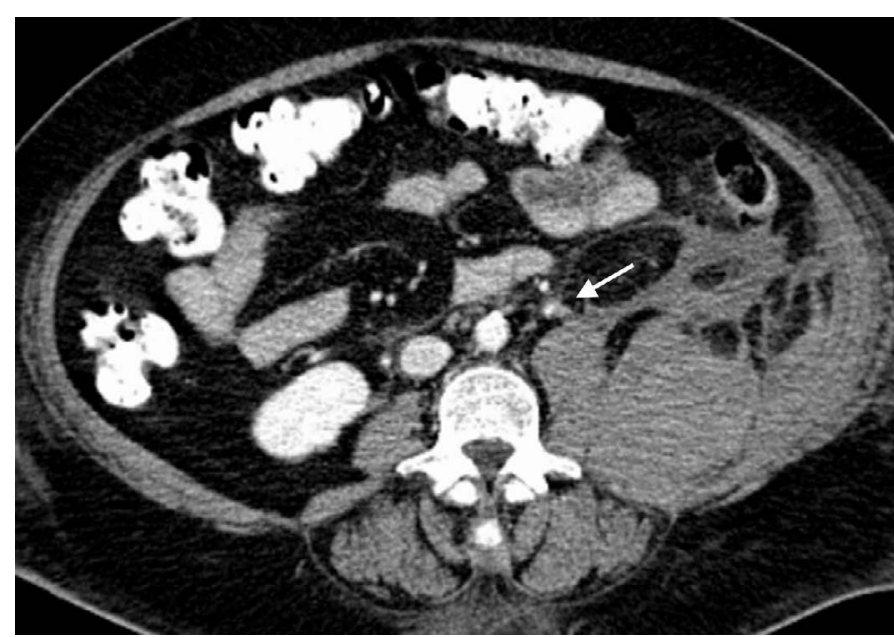

Figure 2. Urgent CT-scan, suspecting an origin of the bleeding on the right lumbar artery.



Figure 3 A: right femoral artery revealed multiple points of bleeding at different branches of the left third lumbar artery. B: Follow-up arteriography showed no further bleeding

\section{Discussion}

Although total hip arthroplasty is generally regarded as safe, an associated mortality with this procedure has been reported $[7,10$ $13,20,21]$.

Several mechanisms of vascular injuries have been described after total hip surgery including perforation of a vessel by a direct trauma (retractors, perioperative manoeuvres, etc.), damage with the heat produced during the polymerisation of the polimethylmethacrylate and the creation of a false aneurism or arteriovenous fistula induced by repeated local trauma [3]. Retroperitoneal haematoma is a rare entity [6]. Any organ or retroperitoneal structure could be the cause of the bleeding but a small number of retroperitoneal haematomas are considered idiopathic or spontaneous [19].

There is a paper [22] of one patient who developed a retroperitoneal bleeding after spinal anesthesia using 22-gauge with the paramedian approach. Opposite to our clinical case, the patient complained of back pain radiating to her left calf, with weakness of the quadriceps muscle four hours after the surgery. In our clinical case the pain and sensation of abdominal fullness started 5 days after the surgery.

The presentation of a retroperitoneal haematoma is normally acute with abdominal pain that can be accompanied by shock signs. Sometimes it can mimic the clinic of an abdominal tumour, like a mass compressing neighbouring structures [23].

The sensation of abdominal fullness associated to an elevated leucocyte count like appendicitis or diverticulitis has also being described. Retroperitoneal haematoma has been called the "left- 
sided appendicitis" by Imdahl et al. [24]. In patients operated and under antithrombotic therapy [25] presenting abdominal pain after the surgery, we need to perform a complete evaluation to reject the existence of a retroperitoneal haematoma.

A contrast-enhanced CT scan is regarded as the most sensitive diagnostic modality in revealing the bleeding site and identifying any underlying pathology [26]. Ultrasonography is less sensitive than CT in detecting renal pathology but may be used as an alternative for following up conservatively-treated retroperitoneal haematomas, as it is a rapid, easily available, cost-effective and radiation-free modality.

Angiography may have both a diagnostic and a therapeutic role in patients with retroperitoneal haemorrhage. Apart from delineating any ruptured vessels, it allows for selective arterial embolization during the same procedure. Embolization reduces the risk of complications such ischemia of surrounding structures [3].

In a centre where arteriography is not available, physicians need to be aware of the clinical signs of retroperitoneal haematoma: abdominal pain, shock signs, mimic the clinic of an abdominal tumour, abdominal fullness associated to an elevated leucocyte count like appendicitis or diverticulitis... To treat the case physicians, need to perform blood transfusions and try to control the bleeding. In case they cannot control it, they need to choose between performing a surgery or transfer the patient to a hospital where they can control the bleeding performing arteriography embolization.

\section{Conclusion}

Total hip arthroplasty is a safe procedure but we need to be alert in operated patients under antithrombotic therapy who present abdominal pain after the surgery and perform a careful evaluation. This case emphasizes the role of minimally invasive techniques, such as arterial embolization, in achieving a positive outcome.

\section{Disclosure form}

No benefits in any form have been received or will be received from a commercial party related directly or indirectly to the subject of this article. No funds were received in support of this study.

\section{References}

1. Maravic M, Landais P (2006) Usefulness of a national hospital database toevaluate the burden of primary joint replacement for coxarthrosis andgonarthrosis in patients aged over 40 years. Osteoarthritis Cartilage 14: 612-615. [Crossref]

2. Touiti D, Zrara I, Ameur A, al Bouzidi A, Beddouch A, et al. (2001) [Spontaneous perirenal hematomas. Report of 3 cases]. Ann Urol (Paris) 35: 319-322. [Crossref]

3. Barriga A, Valenti Nin JR, Delgado C, Bilbao JJ (2001) Therapeutic embolisation for postoperative haemorrhage after total arthroplasty of the hip and knee. J Bone Joint Surg Br 83: 90-92. [Crossref]

4. Austin MS, Klein GR, Sharkey PF, Hozack WJ, Rothman RH (2004) Late sciatic nerve palsy caused by hematoma after primary total hip arthroplasty. J Arthroplasty 19: 790792. [Crossref]

5. Pouliot MA, Lee KB, Goodman SB (2009) Retroperitoneal hematoma: an unusual cause of pain after total hip arthroplasty. J Arthroplasty 24: 1144. [Crossref]
6. Suarez G, Valera Z, Gomez MA, Docobo F, Alamo JM (2005) Hematoma retroperitoneal espontáneo como causa de dolor abdominal y shock hemorrágico Cir Esp 78: 328-330.

7. Zhang JQ, Fielding JR, Zou KH (2002) Etiology of spontaneous perirenal hemorrhage a meta-analysis. J Urol 167: 1593-1596. [Crossref]

8. Reig Ruiz C, Tremps Velasquez E, Margarit Creixell C, Vila Barja J, Palacio EV, et al. (1992) Wunderlich syndrome secondary to the rupture of an aneurysm of the renal artery. Review of the literature. Arch Esp Urol 45: 417-420. [Crossref]

9. Machuca Santa Cruz J, Julve Villalta E, Galacho Bech A, et al. (1999) Spontaneous retroperitoneal hematoma: our experience. Actas Urol Esp 23: 43-50. [Crossref]

10. Nguan C, Leone E (2002) A case of spontaneous perirenal hemorrhage secondary to polyarteritis nodosa. Can J Urol 9: 1704-1706. [Crossref]

11. Hidalgo J, Crego M, Montlleo M, de la Torre P, Bover J, et al. (2005) Embolization of a bleeding aneurysm in a patient with spontaneous perirenal haematoma due to polyarteritis nodosa. Arch Esp Urol 58: 694-697. [Crossref]

12. Mrug M, Mishra PV, Lusane HC, Cunningham JM, Alpert MA (2002) Hemothorax and retroperitoneal hematoma after anticoagulation with enoxaparin. South Med J 95: 936-938. [Crossref]

13. Chan TY (2009) Life-threatening retroperitoneal bleeding due to warfarin-drug interactions. Pharmacoepidemiol Drug Saf 18: 420-422. [Crossref]

14. Ernits M1, Mohan PS, Fares LG 2nd, Hardy H 3rd (2005) A retroperitoneal bleed induced by enoxaparin therapy. Am Surg 71: 430-433. [Crossref]

15. Mrug M, Mishra PV, Lusane HC, Cunningham JM, Alpert MA (2002) Hemothorax and retroperitoneal hematoma after anticoagulation with enoxaparin. South Med J 95: 936-938. [Crossref]

16. Antón E, Marti J (2004) Enoxaparin-associated spontaneous thigh haematoma. Age Ageing 33: 641-642. [Crossref]

17. Kleindienst A, Harvey HB, Mater E, Bronst J, Flack J, et al. (2003) Early antithrombotic prophylaxis with low molecular weight heparin in neurosurgery. Acta Neurochir 145: 1085-1091. [Crossref]

18. Elesber AA, Kent PD, Jennings CA (2001) Compressive neuropathy of the brachial plexus and long thoracic nerve. A rare complication of heparin anticoagulation. Chest 120: 309-311. [Crossref]

19. Al-Khulaiwi A, Razaak FA, El Shair A, Bamehriz F (2000) Idiopathic retroperitoneal hematoma. Ann Saudi Med 20: 270-271. [Crossref]

20. Cinman AC, Farrer J, Kaufman JJ (1985) Spontaneous perinephric hemorrhage in a 65-year-old man. J Urol 133: 829-832. [Crossref]

21. Hyderally HA (2005) Epidural hematoma related to combined spinal-epidural anesthesia in a patient with ankylosing spondylitis receiving aspirin after total hip replacement. Anesth Analg 100: 882-883. [Crossref]

22. Barak M, Fischer D, Gat M, Katz Y (2004) Retroperitoneal hematoma after spinal anesthesia with the paramedian approach. Anesth Analg 98: 851-853. [Crossref]

23. Schuster F, Stosslein F, Steinbach F (2003) Spontaneous rupture of a lumbar artery. A rare etiology of retroperitoneal hematoma. Urologe A 42: 840-844. [Crossref]

24. Imdahl A, Baier P, Ghanem N (2003) [Diverticulosis: the dimensions of a growing problem]. MMW Fortschr Med 145: 28-32. [Crossref]

25. Dabney A, Bastani B (2001) Enoxaparin-associated severe retroperitoneal bleeding and abdominal compartment syndrome: a report of two cases. Intensive Care Med 27: 19541957. [Crossref]

26. Sebastià MC, Pérez-Molina MO, Alvarez-Castells A, Quiroga S, Pallisa E (1997) CT evaluation of underlying cause in spontaneous subcapsular and perirenal hemorrhage. Eur Radiol 7: 686-690. [Crossref]

Copyright: (C2017 Torres-Perez A. This is an open-access article distributed under the terms of the Creative Commons Attribution License, which permits unrestricted use, distribution, and reproduction in any medium, provided the original author and source are credited. 